\title{
A stability theorem for lines in Galois planes of prime order
}

\author{
Tamás Szőnyi and Zsuzsa Weiner * \\ July 18, 2014
}

\begin{abstract}
In this paper we prove that a point set of size less than $\frac{3}{2}(q+1)$ in $\mathrm{PG}(2, q), q$ prime, that has relatively few 0 -secants must contain many collinear points. More precise bounds can be found in Theorem 2.4 .
\end{abstract}

\section{Introduction}

A blocking set $B$ of $\mathrm{PG}(2, q)$ is a set of points intersecting each line in at least one point. Lines intersecting $B$ in exactly one point are called tangents. A point is essential to $B$, if through it there passes at least one tangent of $B$. The blocking set is minimal if all of its points are essential. The smallest examples are lines and a blocking set that does not contain a line is called non-trivial. For a survey on blocking sets, the reader is referred to [10]. When $q$ is a prime, Blokhuis [1] proved an old conjecture by Di Paola.

Result 1.1 (Blokhuis [1]) A non-trivial blocking set in $\mathrm{PG}(2, q)$, q prime, has at least $\frac{3}{2}(q+1)$ points.

If we delete a few say, $\varepsilon$ points from a line, we get a point set intersecting almost all but $\varepsilon q$ lines. After deleting $\varepsilon$ points from a blocking set of size $\frac{3}{2}(q+1)$ (for example, from a projective triangle, see Definition 3.11 in [10])

${ }^{*}$ The authors were partially supported by OTKA NK 67867 and K 81310 grants and by the ERC Grant DISCRETECONT. 
we get at least $\varepsilon \frac{(q-1)}{2} 0$-secant lines. The situation is similar for blocking sets of size at most $2 q$; this follows easily from the result of Blokhuis and Brouwer, see Result 2.1. In general, we cannot expect that the number of points that block the 0 -secants is the number of 0 -secants divided by constant times $q$. To illustrate this, let us consider a blocking set which is the union of parabolas for $q \equiv 1(\bmod 4)$ (see Section 3.3 in $[10])$. Let us delete $\varepsilon$ parabolas completely from this blocking set. Since through each point of a parabola, there passes one tangent line, we will get $\varepsilon q 0$-secants. These lines cannot be blocked by roughly $\varepsilon$ points, since through a point there are at most two tangent lines of a parabola, so one point can block at most $2 \varepsilon$ 0 -secants. This means, that we would need at least $q / 2$ points to block the 0 -secants. We will be interested in the question, when the number of points needed to block the 0 -secants is roughly the number of 0 -secants divided by $q$. If we add these points to our original set, we get a blocking set. Hence we look for a result guaranteeing that a point set having at most $c \varepsilon q 0$-secants must contain a blocking set minus roughly $\varepsilon$ points. Of course, the situation is also interesting when we have less than $q$ skew lines. In this case we wish to prove that the 0 -secants pass through a point. For such a result, formulated in the dual setting, see Proposition 1.5 in [3].

The following old result of Erdős and Lovász can be considered as a stability theorem for lines.

Result 1.2 (Erdős and Lovász, [7]) A point set of size $q$ in a projective plane of order $q$, with less than $\sqrt{q+1}(q+1-\sqrt{q+1}) 0$-secants always contains at least $q+1-\sqrt{q+1}$ points from a line.

Note that the proof of the theorem of Erdős and Lovász can be extended to sets of size less than $q+\sqrt{q}+1$ with a weaker bound on the number of 0 -secants, that is roughly $(q-\sqrt{q})(\sqrt{q}-k)$. The reason that one needs the bound $q+\sqrt{q}+1$ on the size of the set is Bruen's theorem, see [5, 6], namely that the size of a non-trivial minimal blocking set is at least $q+\sqrt{q}+1$.

The main result of the present short note is Theorem 2.4, which can be regarded as an analogue of the Erdös and Lovász theorem (or rather its generalization to $q+k$ ) for Galois planes of prime order. 


\section{Results}

In this section we will improve on the stability theorem of Erdös and Lovász, when the plane is $\mathrm{PG}(2, q), q$ prime. We are going to show that if $B$ is a point set with $|B|<\frac{3}{2}(q+1)$, having at most $\delta=\varepsilon(q+1) 0$-secants, then it contains a huge part of a line. Even though the bound in our main result is not sharp, $\varepsilon$ can even be $c q$, where $c$ is a small constant depending on $|B|$, if $|B|$ is not very close to $\frac{3}{2}(q+1)$.

The following result, which is a consequence of the affine blocking set theorem by Jamison [8], and Brouwer, Schrijver [4], will also be used in our proof.

Result 2.1 (Blokhuis and Brouwer, [2]) Let $B$ be a blocking set in $\mathrm{PG}(2, q)$, $|B|=2 q-s$ and let $P$ be an essential point of $B$. Then there are at least $s+1$ tangents through $P$.

Lemma 2.2 Let $B$ be a point set in $\mathrm{PG}(2, q),|B|<\frac{3}{2}(q+1)$. Assume that there are $\delta$-secants to $B$. Then the total number $\tau$ of tangents of $B$ is at least $(q+1)\left(2 q-|B|-\frac{2 \delta}{q+1}\right)$. Hence there is a point $P$ of $B$ so that there are at least $\frac{2}{3}\left(2 q-|B|-\frac{2 \delta}{q+1}\right)$ tangents through $P$.

Proof. Take a 0 -secant $\ell$ of $B$. If there is no such a line then $B$ is a blocking set and (by Result 2.1) through any essential point of $B$ there pass at least $\frac{1}{2}(q+1)-1$ tangents. Let the points of $\ell$ be denoted by $P_{1}, \ldots, P_{q+1}$ and let $\nu_{i}$ be the number of 0 -secants, $\tau_{i}$ be the number of tangents through $P_{i}$. Looking at $B$ from $P_{i}$ one gets $q-\left(\nu_{i}+\tau_{i}\right) \leq\left(|B|-\tau_{i}\right) / 2$, which implies that $2 \nu_{i}+\tau_{i} \geq 2 q-|B|$. Summing over all $i$ we get that $(q+1)(2 q-|B|) \leq 2 \delta+\tau$, from which $\tau \geq(q+1)\left(2 q-|B|-\frac{2 \delta}{q+1}\right)+1$ follows. On the other hand, if we add up the number of tangents at the points of $B$, we get $\tau$, so there is a point which has at least the average number of tangents.

The following lemma is an easy folklore result in algebraic geometry.

Lemma 2.3 Let $S$ be a set of points in $\mathrm{AG}(2, q)$. Then there exists a nonzero two-variable polynomial of degree at most $\sqrt{2|S|}-1$, so that it vanishes at every point of $S$.

Proof. Each point $(u, v) \in S$, gives a linear equation for the coefficients of the desired polynomial $p$. Hence we have a homogeneous system of $|S|$ such 
linear equations. When $\operatorname{deg}(p) \geq \sqrt{2|S|}-1$, then the number of coefficients is larger than $|S|$, so we have a non-trivial solution.

The proof of our main theorem is motivated by [1] and [9].

Theorem 2.4 Let $B$ be a set of points of $\mathrm{PG}(2, q), q=p$ prime, with at most $\frac{3}{2}(q+1)-\beta$ points. Suppose that the number $\delta$ of 0 -secants is less than $\left(\frac{2}{3}(\beta+1)\right)^{2} / 2$. Then there is a line that contains at least $q-\frac{2 \delta}{q+1}$ points.

Proof. Choose the coordinate system in such a way that $(\infty)$ is a point of $B$ with at least $\frac{2}{3}\left(2 q-|B|-\frac{2 \delta}{q+1}\right)$ tangents, one of them be the line at infinity. Let $U=\left\{\left(a_{i}, b_{i}\right): i=1, \ldots,|B|-1\right\}$ be the affine part of $B$. The 0 -secants of $B$ can be written as $Y=m_{j} X+c_{j}, j=1, \ldots, \delta$. Consider the polynomial $a(x, y)$ of the smallest degree $\Delta$, which vanishes at the points $\left(c_{j}, m_{j}\right), j=1, \ldots, \delta$. By Lemma $2.3, \Delta \leq \sqrt{2 \delta}-1$. Now write up the polynomial

$$
H(X, Y)=\left(\prod\left(X+a_{i} Y-b_{i}\right)\right) a(X, Y) .
$$

The first product is the Rédei polynomial of $U$. This polynomial $H$ vanishes for every $(x, y)$, hence it can be written as

$$
H(X, Y)=\left(X^{q}-X\right) f(X, Y)+\left(Y^{q}-Y\right) g(X, Y),
$$

where $\operatorname{deg}(f), \operatorname{deg}(g) \leq|B|-1-q+\Delta$. As in Blokhuis [1], consider the terms of highest degree of this equation and substitute $Y=1$ in it. Then we get a polynomial equation

$$
h^{*}(X)=\left(\prod\left(X+a_{i}\right)\right) a^{*}(X)=X^{q} f^{*}(X)+g^{*}(X),
$$

where $X^{q} \nmid g^{*}(X)$. We may suppose that $f^{*}$ and $g^{*}$ are coprime, since otherwise we could divide by their greatest common divisor and obtain an equation of the same type with smaller degrees. Denote by $s$ the maximum of the degrees of $f^{*}$ and $g^{*}$ after this division. The roots of $h^{*}(X)$ in $\operatorname{GF}(q)$ are also roots of $X f^{*}(X)+g^{*}(X)$. The multiple roots of $h^{*}(X)$ in $\operatorname{GF}(q)$ are also roots of $X^{q}\left(f^{*}(X)\right)^{\prime}+\left(g^{*}(X)\right)^{\prime}$. The roots not in $\mathrm{GF}(q)$ are roots of $a^{*}(x)$. Hence

$$
h^{*}(X) \mid\left(X f^{*}(X)+g^{*}(X)\right)\left(\left(f^{*}(X)\right)^{\prime} g^{*}(X)-\left(g^{*}(X)\right)^{\prime} f^{*}(X)\right) a^{*}(X) .
$$


If the polynomial on the right hand side of (1) is non-zero, then comparing the degrees gives $q+s \leq s+1+2 s-2+\Delta$, that is $s \geq(q+1-\Delta) / 2$. Since $s \leq|B|-1-q+\Delta$, then $|B| \geq \frac{3}{2}(q+1)-\frac{3}{2} \Delta$, which is a contradiction.

The third term on the right hand side of (1) cannot be the zero polynomial, since the terms of highest degree of $a(X, Y)$ form a homogeneous polynomial and so $(Y-1)$ cannot be a factor of it.

If the first term on the right hand side of $(1)$ is the zero polynomial then $h^{*}(X)$ is divisible by $\left(X^{q}-X\right)$. Since $a^{*}(X)$ has degree at most $\Delta$, the remaining $q-\Delta$ factors of $\left(X^{q}-X\right)$ must arise from the product $\prod\left(X+a_{i}\right)$. Geometrically this would imply that through the point $(\infty)$ there pass at most $\Delta+1$ tangents, which contradicts the choice of $(\infty)$. (Here we use that $\Delta+1<\frac{2}{3}\left(2 q-|B|-\frac{2 \delta}{q+1}\right)$.)

If the second term is zero, then, since $f^{*}$ and $g^{*}$ are coprime, $f^{*}(X) \mid\left(f^{*}(X)\right)^{\prime}$ and similarly $g^{*}(X) \mid\left(g^{*}(X)\right)^{\prime}$. Hence $\left(f^{*}(X)\right)^{\prime}=\left(g^{*}(X)\right)^{\prime}=0$. For $q=p$ prime, it implies that either $|B| \geq 2 q+1-\Delta$ (which is not possible by our upper bound on $|B|)$ or $a X^{q}+b$ divides $h^{*}(X)$. Since $a X^{q}+b=(a X+b)^{q}$ and at most $\Delta$ of these factors can come from $a^{*}(X)$, then there is a line $\ell$ (through $(\infty)$ ) that contains at least $q+1-\Delta$ points of $B$. Finally, assume that $|\ell \cap B|=q+1-k, k \leq \Delta$. Then the 0 -secants pass through the $k$ missing points of $\ell$. Since $|B| \leq \frac{3}{2} q+1-\frac{3}{2} \Delta$ then the number of 0 -secants is at least $k\left(q-\left(\frac{3}{2} q+1-\frac{3}{2} \Delta-q-1+k\right)\right) \leq \frac{1}{2} k(q+1)$. Hence $k \leq \frac{2 \delta}{q+1}$.

Let us see now some constructions for sets with few 0-secants not containing a very large collinear subset. Deleting $\varepsilon$ points from a line or a projective triangle yields $\varepsilon q$ or at least $\varepsilon \frac{q-1}{2} 0$-secants, respectively. In the former case the number of deleted points is $\frac{\delta}{q}$, in the latter case it is roughly $\frac{2 \delta}{q}$. The constructions below can be regarded as generalizations of the Rédei-Megyesi construction for blocking sets (see Theorem 3.10 in [10]). In the constructions we use standard notation: affine points are denoted as $(u, v)$, ideal point as $(m)$ or $(\infty)$.

Construction 2.5 Assume that $3 \mid q-1$ and let $H$ be a subgroup of $G F(q)^{*}$, $|H|=\frac{q-1}{3}$. Furthermore, let $B$ be the set of size $q+2$, where

$B=\{(0, h) \mid h \in H\} \cup\{(h, 0) \mid h \in H\} \cup\{(h) \mid h \in H\} \cup\{(0,0)\} \cup(0) \cup(\infty)\}$.

Then the number of 0 -secants to $B$ is $\frac{2}{9}(q-1)^{2}$. Add $k<\frac{q+17}{6}$ ideal points not in $B$ to obtain $B^{\prime}$. Then the total number of 0 -secants to $B^{\prime}$ is $\left(\frac{2}{3}(q-\right.$ 1) $-k) \frac{1}{3}(q-1)$. 
The sets constructed above have less than $\left(\frac{3}{2}(q+1)-\left|B^{\prime}\right|\right) \frac{q-1}{2} 0$-secants (this is what we would get for a set contained in a projective triangle) and are not contained in a projective triangle.

In general, one could choose a multiplicative subgroup $H$ (of size $\frac{q-1}{t}$ ) from the line $Y=0, s$ cosets of $H$ from the line $X=0$, and the same $s$ cosets from the ideal line. For example, when $t=2 s,\left|B^{\prime}\right|=q+2+\frac{q-1}{2 s}$ and the number of 0 -secants is roughly $\frac{q-1}{2}\left(\frac{q-1}{2}-\frac{q-1}{2 s}\right)$, which is the same as one could get by deleting $\left(\frac{q-1}{2}-\frac{q-1}{2 s}\right)$ appropriate points from a projective triangle. For $t>2$ the cosets can be chosen in such a way that the set is not contained in a projective triangle.

Construction 2.6 Let $A$ and $B$ be less than $p$ and let $B^{*}$ be a the following set.

$B^{*}=\{(1, a) \mid 0 \leq a \leq A\} \cup\{(0,-b) \mid 0 \leq b \leq B\} \cup\{(\infty)\} \cup\{(c) \mid 0 \leq c \leq A+B\}$.

Then $B^{*}$ has $2(A+B)+4$ points and the total number of 0 -secants to $B^{*}$ is $(q-1-A-B)(q-A-B-2)$.

One can modify this construction and delete some points $(c)(0 \leq c \leq$ $A+B)$ which are on many tangents of $B^{*}$. To be more concrete choose $\alpha$ and $\beta$, so that $\alpha \leq A+B-\beta$. Let

$$
B^{* *}=\left\{\left(B^{*} \cap A G(2, p)\right) \cup(\infty) \cup\{(c) \mid \alpha \leq c \leq A+B-\beta\} .\right.
$$

Then $\left|B^{* *}\right|=2(A+B)+4-\alpha-\beta$ and there are $(q-1-A-B)(q-$ $A-B-2)+\frac{\alpha}{2}(2 q+\alpha-3-2(A+B))+\frac{\beta}{2}(2 q+\beta-3-2(A+B)) 0$-secants.

For $A=B=\frac{p}{4}, B^{*}$ has size $p+4$ and the number of 0 -secants is roughly $\frac{p^{2}}{4}$, which is the number of 0 -secants of the a point set obtained by keeping these many points of a projective triangle, but this set obviously cannot be embedded in a projective triangle. One can also combine Constructions 2.6 and 2.5 by replacing arithmetic by geometric progressions. For example, if $t=5, H$ is a multiplicative subgroup and $\omega$ generates $G / H$ then

$$
\begin{aligned}
B^{* * *}= & \{(0, h) \mid h \in H \cup \omega H\} \cup\left\{(h, 0) \mid h \in H \cup \omega^{-1} H\right\} \cup \\
& \left.\left\{(h) \mid h \in H \cup \omega H \cup \omega^{2} H\right\} \cup\{(0,0)\} \cup(0) \cup(\infty)\right\}
\end{aligned}
$$

has $3+\frac{7}{5}(q-1)$ points and $\delta=\frac{2}{25}(q-1)^{2} 0$-secants. By deleting the points (h), $h \in \omega^{2} H$ we get a set of size $3+\frac{6}{5}(q-1)$ points which has $\delta=\frac{4}{25}(q-1)^{2}$. 
All the examples given above can be obtained from a blocking set contained in the union of three lines by deleting quite a few points. In some cases they have less 0-secants than a set of the same size contained in the projective triangle. The examples also show that we cannot expect $\frac{\delta}{q+1}$ missing points from a line in Theorem 2.4 .

Remark 2.7 1) In the case corresponding to the Erdős and Lovász theorem, that is when $|B|=q$, we can allow roughly $\frac{q^{2}}{18}$ 0-secants to guarantee a collinear subset of size at least $\frac{8 q}{9}$ in $B$. The bound $\frac{q^{2}}{18}$ can most likely be improved but we do need an upper bound of the form $c q^{2},\left(c \leq \frac{1}{2}\right)$ on the number of 0 -secants as shown by the constructions above.

2) If a set $B$ has size $|B|=c q$ for some $c<1$ then the number of 0 secants is at least $(1-c) q(q+1)$. This can be seen by counting 0 -secants through points of a fixed 0 -secant. Hence our theorem gives a non-trivial bound only if $(1-c) q(q+1)<\left(\frac{2}{3}(\beta+1)\right)^{2} / 2$, where $\beta=\left(\frac{3}{2}-c\right) q$. Roughly speaking, this gives that for a fixed $c<1$, the value of $\delta$ has to be smaller than $\left(1-\frac{2}{3} c\right)^{2} q^{2} / 2$, which gives the equation $4 c^{2}+6 c-9=0$ for the critical value of c. The positive root of the equation is $\frac{-3+\sqrt{45}}{4} \approx 0.927$. So our result gives a non-trivial stability theorem also for sets of size $c q, 1>c>\frac{-3+\sqrt{45}}{4} \approx 0.927$.

\section{References}

[1] A. Blokhuis, On the size of a blocking set in $\mathrm{PG}(2, p)$, Combinatorica 14 (1994), 273-276.

[2] A. Blokhuis, A. E. Brouwer, Blocking sets in Desarguesian projective planes, Bull. London Math. Soc. 18 (1986), 132-134.

[3] A. Blokhuis, A. E. Brouwer, T. SzŐNyi, Covering all points except one, J. Alg. Combin. 32 (2010), 59-66.

[4] A. E. Brouwer, A. Schrijver, The blocking number of an affine space, J. Comb. Theory Ser. A 24 (1978), 251-253.

[5] A. A. Bruen, Baer subplanes and blocking sets, Bull. Amer. Math. Soc. 76 (1970), 342-344.

[6] A. A. Bruen, Blocking sets in finite projective planes, SIAM J. Appl. Math. 21 (1971), 380-392. 
[7] P. ERdős, L. Lovász, Problems and results on 3-chromatic hypergraphs and some related questions, in: Infinite and finite sets (Colloq., Keszthely, 1973; dedicated to P. Erdős on his 60th birthday), Vol. II, pp. 609-627, Colloq. Math. Soc. János Bolyai, Vol. 10, North-Holland, Amsterdam, 1975.

[8] R. E. Jamison, Covering finite fields with cosets of subspaces, J. Comb. Theory Ser. A 22 (1977), 253-266.

[9] T. SzŐNYI, Around Rédei's theorem, Discrete Math. 208/9 (1999), 557575.

[10] T. SzŐnYi, A. GÁcs, Zs. Weiner, On the spectrum of minimal blocking sets, J. Geometry 76 (2003), 256-281.

Authors address:

Tamás Szőnyi, Zsuzsa Weiner

Department of Computer Science, Eötvös Loránd University,

H-1117 Budapest, Pázmány Péter sétány 1/C, HUNGARY

e-mail: szonyi@cs.elte.hu, weiner@cs.elte.hu

Tamás Szőnyi,

Computer and Automation Research Institute of the Hungarian Academy of Sciences

H-1111 Budapest, Lágymányosi út 11, HUNGARY

Zsuzsa Weiner

Prezi.com

H-1088 Budapest, Krúdy Gyula utca 12, HUNGARY

e-mail: zsuzsa.weiner@prezi.com 